\title{
Cosine modulation of the unpolarized pion cross section at HERMES
}

\author{
Francesca Giordano* \\ (on behalf of the HERMES Collaboration) \\ DESY, Hamburg \\ E-mail: francesca.giordano@desy.de \\ Rebecca Lamb \\ University of Illinois \\ E-mail: rlamb2eillinois.edu
}

\begin{abstract}
An extraction of azimuthal $\cos \phi_{h}$ and $\cos 2 \phi_{h}$ modulations of pion distributions in unpolarized semi-inclusive deep-inelastic scattering has been performed at HERMES by means of a multidimensional $\left(x, y, z, P_{h \perp}\right)$ unfolding procedure to disentangle the azimuthal dependence of the cross section from instrumental and radiative contributions. Results are presented for hydrogen and deuterium targets and separately for positively and negatively charged pions to access flavor dependent information about quark intrinsic transverse momenta and spin-orbit correlations.
\end{abstract}

XVIII International Workshop on Deep-Inelastic Scattering and Related Subjects, DIS 2010 April 19-23, 2010

Firenze, Italy

\footnotetext{
${ }^{*}$ Speaker.
} 


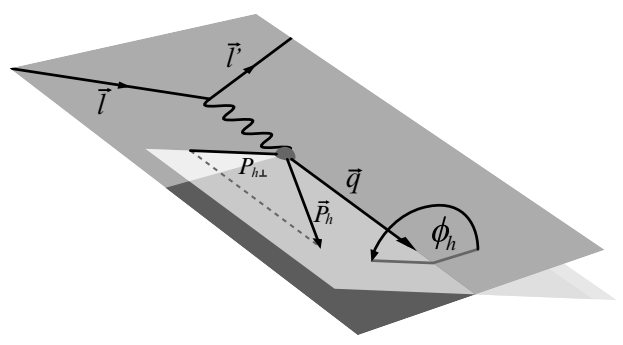

Figure 1: Definition of the azimuthal angle $\phi_{h}$ between the scattering plane (dark grey) and the hadron production plane (light grey).

\section{Introduction}

In lepton-nucleon Deep-Inelastic Scattering (DIS) the structure of the nucleon is probed by the interaction of a high energy lepton with a target nucleon, via the exchange of one virtual boson. If at least one of the produced hadrons is detected in coincidence with the scattered lepton, the reaction is called Semi-Inclusive Deep-Inelastic Scattering (SIDIS):

$$
l(\mathbf{k})+N(\mathbf{P}) \rightarrow l^{\prime}\left(\mathbf{k}^{\prime}\right)+h\left(\mathbf{P}_{h}\right)+X\left(\mathbf{P}_{X}\right)
$$

where $l\left(l^{\prime}\right)$ is the incident (scattered) lepton, $N$ is the target nucleon, $h$ is a detected hadron, $X$ is the target remnant and the quantities in parentheses are the corresponding four-momenta.

If the cross section is unintegrated over the hadron momentum component transverse to the virtual photon direction $P_{h \perp}$ (fig. 1), an azimuthal dependence in the outgoing hadron direction appears [1]:

$$
\begin{aligned}
\frac{d \sigma}{d x d y d z d P_{h \perp}^{2} d \phi_{h}}= & \frac{\alpha^{2}}{x y Q^{2}}\left(1+\frac{\gamma^{2}}{2 x}\right)\left\{A(y) F_{U U, T}+B(y) F_{U U, L}+\right. \\
& \left.C(y) \cos \phi_{h} F_{U U}^{\cos \phi_{h}}+B(y) \cos 2 \phi_{h} F_{U U}^{\cos 2 \phi_{h}}\right\},
\end{aligned}
$$

where $\phi_{h}$ is the azimuthal angle of the hadron plane around the virtual-photon direction (fig. 1). Here $Q^{2}$ and $y$ are respectively the negative squared four-momentum and the fractional energy of the virtual photon, $x$ is the Bjorken scaling variable and $z$ is the fractional energy of the produced hadron. The subscripts $U U$ stand for Unpolarized beam and target, $T(L)$ indicates the Transverse (Longitudinal) polarization of the virtual photon, $\alpha$ is the electromagnetic coupling constant, $\gamma=$ $2 M x / Q$ with $M$ the target mass, $A(y) \sim\left(1-y+1 / 2 y^{2}\right), B(y) \sim(1-y)$ and $C(y) \sim(2-y) \sqrt{1-y}$.

Among the possible ones, two mechanisms are expected to give important contributions to the azimuthal dependence of the unpolarized cross section in the hadron transverse momentum range accessed at HERMES. The first one is called the Cahn effect [2], a pure kinematic effect where the azimuthal modulations are generated by the non-zero intrinsic transverse motion of quarks. In the second mechanism, the Boer-Mulders effect [3], $\cos \phi_{h}$ and $\cos 2 \phi_{h}$ modulations originate from the coupling of the quark intrinsic transverse momentum and intrinsic transverse spin, a sort of spin-orbit effect. 


\subsection{The HERMES experiment}

The results presented here are extracted from data collected at HERMES in the 2000-2007 data taking periods. The fixed-target HERMES experiment ran for more than 10 years until 2007 at the lepton-proton storage ring of HERA at DESY that could operate with either electrons or positrons.

The HERMES spectrometer [4] was a forward-angle instrument consisting of two symmetric halves above and below the horizontal plane defined by the lepton beam pipe. It was characterized by very high efficiency (around $98-99 \%$ ) in lepton-hadron separation, provided by a transition radiation detector, a preshower scintillation counter and an electromagnetic calorimeter. In addition, a dual-radiator Ring-Imaging CHerenkov (RICH) detector provided hadron identification for momenta above $2 \mathrm{GeV} / \mathrm{c}$.

\section{Multidimensional extraction and unfolding procedure}

To access flavor dependent information about the azimuthal dependent structure functions $F_{U U}^{\cos \phi_{h}}$ and $F_{U U}^{\cos 2 \phi_{h}}$ defined in Eq. (1.3), the $\left\langle\cos n \phi_{h}\right\rangle$-moments (= $\frac{\int \cos n \phi_{h} \sigma d \phi_{h}}{\int \sigma d \phi_{h}}$, with $\left.n=1,2\right)$ were measured for hadrons identified by the RICH as pions.

The extraction of these cosine moments from data is challenging because they couple to a number of experimental sources of azimuthal modulations, e.g. detector geometrical acceptance and higher-order QED effects (radiative effects). Moreover, in the typical case, the event sample is binned only in one variable (1-dimensional analysis), and integrated over the full range of all the other ones, but the mentioned structure functions and the instrumental spurious contributions depend on all the kinematic variables $x, y, z$ and $P_{h \perp}$ simultaneously. In order to account for correlations between the physical modulations and these spurious contributions, a multi-dimensional analysis is needed where the event sample is binned simultaneously in all the relevant variables ${ }^{1}$.

The unfolding procedure [6] described below is used to correct the extracted cosine moments for radiative and detector smearing. A detailed Monte Carlo simulation of the experimental apparatus is used to define the Smearing matrix $S(i, j)$, which provides the probability that an event generated in the Born bin $j$, is observed in a different measured bin $i$, due to experimental distortions. The unfolding algorithm relates the unknown Born yield distribution $n_{B}(j)$ to the measured yield distribution $n(i)$ via the Smearing matrix:

$$
n(i)=\sum_{j=1}^{n_{b}} S(i, j) n_{B}(j)+n_{b g}(i),
$$

where $n_{b}$ is the total number of bins and $n_{b g}(i)$ contains the events smeared into the measured sample from outside the acceptance (evaluated from a Monte Carlo simulation).

Assuming a non-singular $S(i, j)$, Eq.(2.1) directly provides the Born yields:

$$
n_{B}(j)=\sum_{i=1}^{n_{b}} S^{-1}(j, i)\left[n(i)-n_{b g}(i)\right]
$$

Since the unfolding mixes different bins contents, the unfolded Born yields are statistically correlated, and the extraction of cosine moments from them requires a generalized least-squares

\footnotetext{
${ }^{1}$ For a more detailed discussion about mono- and multi-dimensional analysis see [5].
} 


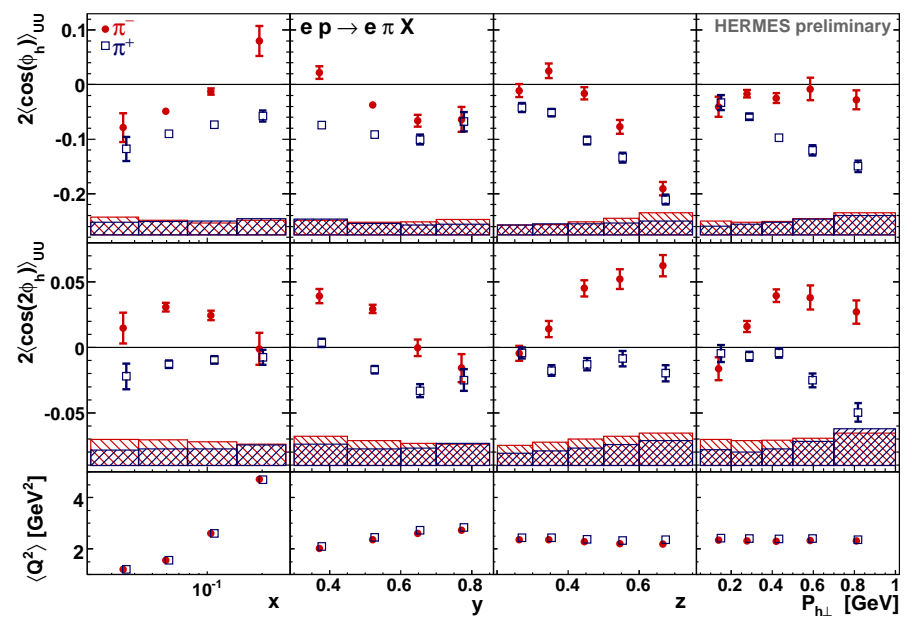

Figure 2: $\cos \phi_{h}$ (upper panel) and $\cos 2 \phi_{h}$ (lower panel) moments for positive (open squares) and negative (closed circles) pions extracted with a hydrogen target, projected versus the kinematic variables $x, y, z$ and $P_{h \perp}$.

fit which takes into account the non-diagonal covariance matrix. A linear regression can then be performed to linearize the problem and extract one $\left(\left\langle\cos \phi_{h}\right\rangle,\left\langle\cos 2 \phi_{h}\right\rangle\right)$ moment pair for each kinematic bin, which represents fully differential results. The moment dependence on a single variable can be obtained integrating the fully differential results (weighted by the cross section) over the other kinematic variables.

\section{Results}

The cross section unintegrated over pion transverse momentum gives access to new exciting aspects of the nucleon structure, which are currently under intense theoretical investigations. Because they are experimentally challenging, very few measurements have been performed to date $([7,8,9,10,11,12,13])$.

The azimuthal moments extracted with a hydrogen target are shown in figure 2 for positive (open squares) and negative (closed circles) pions, projected in the relevant kinematic variables. The $\left\langle\cos \phi_{h}\right\rangle$ moments (upper panel) are found to be sizable and negative for positive pions, the signal increases with $P_{h \perp}$ and with the pion energy fraction $z$. The results for negatively charged pions exhibit similar features as the positively charged pions, but the signal size is significantly lower. The $\cos 2 \phi_{h}$ moments (lower panel) are found to be slightly negative for positive pions, while negative pions show positive $\cos 2 \phi_{h}$ modulations.

The cosine modulations extracted with a deuterium target show similar features as the hydrogen ones, as shown in figure 3.

For the first time in SIDIS experiments, cosine azimuthal modulations have been extracted for identified pions, giving access to flavor dependent informations. Although there exist still no theoretical model that can fully describes the $\cos \phi_{h}$ and $\cos 2 \phi_{h}$ modulations measured at HERMES, the different results for positive and negative pions, and in particular the opposite sign measured in the $\left\langle\cos 2 \phi_{h}\right\rangle$ moments, can be considered an evidence of a non-zero Boer-Mulders effect $[14,15,16]$. 


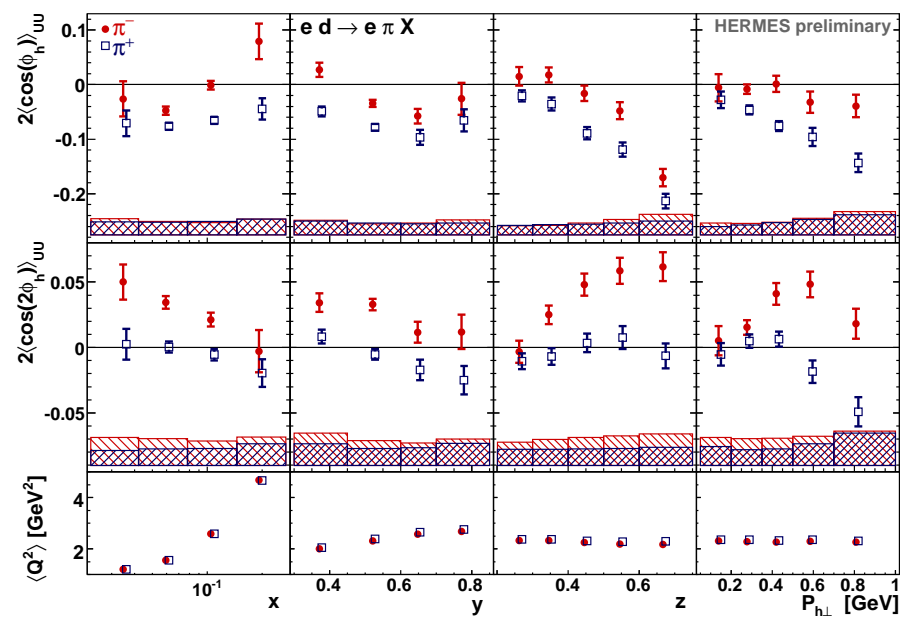

Figure 3: $\cos \phi_{h}$ (upper panel) and $\cos 2 \phi_{h}$ (lower panel) moments for positive (open squares) and negative (closed circles) pions extracted with a deuterium target, projected versus the kinematic variables $x, y, z$ and $P_{h \perp}$.

\section{References}

[1] A. Bacchetta and others, JHEP 02, 093 (2007)

[2] N. R. Cahn, Phys. Lett. B78, 269 (1978)

N. R. Cahn, Phys. Rev. D40,3107 (1989)

[3] D. Boer and P. J. Mulders, Phys. Rev. D57, 5780 (1998)

[4] K. Ackerstaff and others, Nucl. Instrum. Meth. A417, 230 (1998)

[5] F. Giordano, in proceeding of Transversity 2008 Workshop, World Scientific, 177 (2008)

[6] G. Cowan, Statistical Data Analysis, Clarendon Press, Oxford, UK (1998)

[7] G. Kafer, in proceeding of Transversity 2008 Workshop, World Scientific, 169 (2008)

[8] EMC Collaboration, J. J. Aubert et al., Phys. Lett. B130:118, 1983

EMC Collaboration, M. Arneodo et al., Z. Phys. C34:277,1987

[9] ZEUS Collaboration, M. Derrick et al., Z. Phys. C25:361, 1991

ZEUS Collaboration, J. Breitweg et al., Phys. Lett.B481:199, 2000

[10] E665 Collaboration, M. R. Adam et al., Phys. Rev. D48:5057, 1993

[11] L. Y Zhu et al, FNAL E866/NuSea Collaboration, Phys, Rev. Lett. 99:082301, 2007

[12] S. Falciano et al., NA10 Collaboration, Z. Phys. C31:513, 1986 M. Guanziroli et al., NA10 Collaboration, Z. Phys. C37:545, 1988

[13] J. S. Conway et al., E615 Collaboration, Phys. Rev. D39:92, 1989 J.G. Heinrich et al., E615 Collaboration, Phys. Rev. D44:1909,1991

[14] L. P. Gamberg, proceedings of Spin 2004 Conference, 334 Trieste/Mainz (2004)

[15] V. Barone, A. Prokudin and B. Q. Ma, Phys. Rev. D78, 045022 (2008)

[16] B. Zhang and others, Phys.Rev.D78, 034035 (2008) 